\title{
Threshold effects in non-dynamic panels: Estimation, testing, and inference
}

\author{
Bruce E. Hansen ${ }^{1}$ \\ Department of Economics, University of Wisconsin, Social Science Building, 1180 Observatory Drive, \\ Madison, WI 53706, USA
}

Received 1 June 1997; received in revised form 1 March 1999; accepted 1 April 1999

\begin{abstract}
Threshold regression methods are developed for non-dynamic panels with individualspecific fixed effects. Least squares estimation of the threshold and regression slopes is proposed using fixed-effects transformations. A non-standard asymptotic theory of inference is developed which allows construction of confidence intervals and testing of hypotheses. The methods are applied to a 15-year sample of 565 US firms to test whether financial constraints affect investment decisions. (C) 1999 Elsevier Science S.A. All rights reserved.
\end{abstract}

JEL classification: $\mathrm{C} 33 ; \mathrm{C} 12 ; \mathrm{C} 13$

Keywords: Threshold regression; Panel data; Liquidity constraints; Investment; Nonstandard distribution

\section{Introduction}

Are regression functions identical across all observations in a sample, or do they fall into discrete classes? This question may be addressed using threshold

\footnotetext{
${ }^{1}$ Homepage: http://www.ssc.wisc.edu/ bhansen/.

E-mail address: bhansen@ssc.wisc.edu (B.E. Hansen)
} 
regression techniques. Threshold regression models specify that individual observations can be divided into classes based on the value of an observed variable. Despite their intuitive appeal, econometric techniques have not been well developed for threshold regression.

This paper introduces econometric techniques appropriate for threshold regression with panel data. Least squares estimation methods are described. An asymptotic distribution theory is derived which is used to construct confidence intervals for the parameters. A bootstrap method to assess the statistical significance of the threshold effect is also described. The methods are similar to those developed in earlier work by the author (Hansen, 1996, 1999).

The methods are used to investigate whether financial constraints affect the investment practices of firms. The classical theory of the firm suggests that financing should have no allocative effects (e.g., the Modigliani-Miller theorem). Investment decisions should only be based on the marginal $Q$ of a specific project, since banks will be willing to extend finance. In the context of imperfect information, external financing may be limited, and debtconstrained firms may need to finance investment out of cash flow. If this is the case, investment will be correlated with cash flow for constrained firms. This observation led Fazzari et al. (1988) to divide a sample of US firms into classes based on their degree of financial constraints and estimate the differing effects of cash flow on investment among these classes. Their analysis suffered from two problems. First, they used an endogenous variable (dividend to income ratio) rather than an exogenous variable to form their sample splits. Second, they used an ad hoc method to select their sample splits. We repeat their analysis on an analogous data set using appropriate econometric techniques and find qualitatively similar results.

Other authors have investigated the implications of non-linear $q$ models of investment. Abel and Eberly (1994) propose a model which implies that the response of investment to $q$ may be non-linear in $q$. Abel and Eberly (1996) use panel data to estimate a similar model, and find evidence for non-linearities in the investment function. Barnett and Sakellaris (1998) find similar results using a threshold regression approach. Hu and Schiantarelli (1998) use a switching regression framework to study the same problem. Our paper extends and reinforces this growing literature.

The next section introduces the model and notation. Section 3 discusses estimation by fixed effects. Section 4 outlines our asymptotic theory of inference. A distribution theory is developed for the threshold estimate and the slope coefficients. Section 5 reports the empirical application to firms' investment decisions. Section 6 concludes. Proofs of the asymptotic theory are provided in the appendix. GAUSS programs and data which replicate the empirical work are available from the author's homepage. 


\section{Model}

The observed data are from a balanced ${ }^{2}$ panel $\left\{y_{i t}, q_{i t}, x_{i t}\right.$ : $1 \leqslant i \leqslant n, 1 \leqslant t \leqslant T\}$. The subscript $i$ indexes the individual and the subscript $t$ indexes time. The dependent variable $y_{i t}$ is scalar, the threshold variable $q_{i t}$ is scalar, and the regressor $x_{i t}$ is a $k$ vector. The structural equation of interest is

$$
y_{i t}=\mu_{i}+\beta_{1}^{\prime} x_{i t} I\left(q_{i t} \leqslant \gamma\right)+\beta_{2}^{\prime} x_{i t} I\left(q_{i t}>\gamma\right)+e_{i t} .
$$

where $I(\cdot)$ is the indicator function. An alternative intuitive way of writing (1) is

$$
y_{i t}= \begin{cases}\mu_{i}+\beta_{1}^{\prime} x_{i t}+e_{i t}, & q_{i t} \leqslant \gamma, \\ \mu_{i}+\beta_{2}^{\prime} x_{i t}+e_{i t}, & q_{i t}>\gamma .\end{cases}
$$

Another compact representation of (1) is to set

$$
x_{i t}(\gamma)=\left(\begin{array}{l}
x_{i t} I\left(q_{i t} \leqslant \gamma\right) \\
x_{i t} I\left(q_{i t}>\gamma\right)
\end{array}\right)
$$

and $\beta=\left(\begin{array}{lll}\beta_{1}^{\prime} & \beta_{2}^{\prime}\end{array}\right)^{\prime}$ so that (1) equals

$$
y_{i t}=\mu_{i}+\beta^{\prime} x_{i t}(\gamma)+e_{i t}
$$

The observations are divided into two 'regimes' depending on whether the threshold variable $q_{i t}$ is smaller or larger than the threshold $\gamma$. The regimes are distinguished by differing regression slopes, $\beta_{1}$ and $\beta_{2}$. For the identification of $\beta_{1}$ and $\beta_{2}$, it is required that the elements of $x_{i t}$ are not time invariant. We also assume that the threshold variable $q_{i t}$ is not time invariant. The error $e_{i t}$ is assumed to be independent and identically distributed (iid) with mean zero and finite variance $\sigma^{2}$. The iid assumption excludes lagged dependent variables from $x_{i t}$. It is unclear how to extend the results to allow for dynamic models and/or heteroskedastic errors. The analysis is asymptotic with fixed $T$ as $n \rightarrow \infty$.

\section{Estimation}

\subsection{Least squares estimation}

One traditional method to eliminate the individual effect $\mu_{i}$ is to remove individual-specific means. While straightforward in linear models, the nonlinear specification (1) calls for a more careful treatment. Note that taking

\footnotetext{
${ }^{2}$ It is unknown if the results extend to unbalanced panels.
} 
averages of (1) over the time index $t$ produces

$$
\bar{y}_{i}=\mu_{i}+\beta^{\prime} \bar{x}_{i}(\gamma)+\bar{e}_{i}
$$

where $\bar{y}_{i}=T^{-1} \sum_{t=1}^{T} y_{i t}, \bar{e}_{i}=T^{-1} \sum_{t=1}^{T} e_{i t}$, and

$$
\begin{aligned}
\bar{x}_{i}(\gamma) & =\frac{1}{T} \sum_{t=1}^{T} x_{i t}(\gamma) \\
& =\left(\begin{array}{l}
\frac{1}{T} \sum_{t=1}^{T} x_{i t} I\left(q_{i t} \leqslant \gamma\right) \\
\frac{1}{T} \sum_{t=1}^{T} x_{i t} I\left(q_{i t}>\gamma\right)
\end{array}\right) .
\end{aligned}
$$

Taking the difference between (2) and (3) yields

$$
y_{i t}^{*}=\beta^{\prime} x_{i t}^{*}(\gamma)+e_{i t}^{*}
$$

where

$$
\begin{aligned}
& y_{i t}^{*}=y_{i t}-\bar{y}_{i}, \\
& x_{i t}^{*}(\gamma)=x_{i t}(\gamma)-\bar{x}_{i}(\gamma),
\end{aligned}
$$

and

$$
e_{i t}^{*}=e_{i t}-\bar{e}_{i}
$$

Let

$$
y_{i}^{*}=\left[\begin{array}{c}
y_{i 2}^{*} \\
\vdots \\
y_{i T}^{*}
\end{array}\right], \quad x_{i}^{*}(\gamma)=\left[\begin{array}{c}
x_{i 2}^{*}(\gamma)^{\prime} \\
\vdots \\
x_{i T}^{*}(\gamma)^{\prime}
\end{array}\right], \quad e_{i}^{*}=\left[\begin{array}{c}
e_{i 2}^{*} \\
\vdots \\
e_{i T}^{*}
\end{array}\right]
$$

denote the stacked data and errors for an individual, with one time period deleted. Then let $Y^{*}, X^{*}(\gamma)$ and $e^{*}$ denote the data stacked over all individuals, for example

$$
X^{*}(\gamma)=\left[\begin{array}{c}
x_{1}^{*}(\gamma) \\
\vdots \\
x_{i}^{*}(\gamma) \\
\vdots \\
x_{n}^{*}(\gamma)
\end{array}\right] .
$$

Using this notation, (4) is equivalent to

$$
Y^{*}=X^{*}(\gamma) \beta+e^{*} .
$$


For any given $\gamma$, the slope coefficient $\beta$ can be estimated by ordinary least squares (OLS). That is,

$$
\widehat{\beta}(\gamma)=\left(X^{*}(\gamma)^{\prime} X^{*}(\gamma)\right)^{-1} X^{*}(\gamma)^{\prime} Y^{*} .
$$

The vector of regression residuals is

$$
\hat{e}^{*}(\gamma)=Y^{*}-X^{*}(\gamma) \hat{\beta}(\gamma)
$$

and the sum of squared errors is

$$
\begin{aligned}
& S_{1}(\gamma)=\hat{e}^{*}(\gamma)^{\prime} \hat{e}^{*}(\gamma) \\
& =Y^{*}\left(I-X^{*}(\gamma)^{\prime}\left(X^{*}(\gamma)^{\prime} X^{*}(\gamma)\right)^{-1} X^{*}(\gamma)^{\prime}\right) Y^{*} .
\end{aligned}
$$

Chan (1993) and Hansen (1999) recommend estimation of $\gamma$ by least squares. This is easiest to achieve by minimization of the concentrated sum of squared errors (7). Hence the least squares estimators of $\gamma$ is

$$
\hat{\gamma}=\underset{\gamma}{\operatorname{argmin}} S_{1}(\gamma) \text {. }
$$

It is undesirable for a threshold $\hat{\gamma}$ to be selected which sorts too few observations into one or the other regime. This possibility can be excluded by restricting the search in (8) to values of $\gamma$ such that a minimal percentage of the observations (say, $1 \%$ or $5 \%$ ) lie in each regime.

Once $\hat{\gamma}$ is obtained, the slope coefficient estimate is $\hat{\beta}=\hat{\beta}(\hat{\gamma})$. The residual vector is $\hat{e}^{*}=\hat{e}^{*}(\hat{\gamma})$ and residual variance

$$
\hat{\sigma}^{2}=\frac{1}{n(T-1)} \hat{e}^{*^{\prime}} \hat{e}^{*}=\frac{1}{n(T-1)} S_{1}(\hat{\gamma})
$$

\subsection{Computation issues}

The computation of the least squares estimate of the threshold $\gamma$ involves the minimization problem (8). Since the sum of squared error function $S_{1}(\gamma)$ depends on $\gamma$ only through the indicator functions $I\left(q_{i t} \leqslant \gamma\right)$, the sum of squared error function is a step function with at most $n T$ steps, with the steps occurring at distinct values of the observed threshold variable $q_{i t}$. Thus the minimization problem (8) can be reduced to searching over values of $\gamma$ equalling the (at most $n T$ ) distinct values of $q_{i t}$ in the sample.

To implement the minimization, the following approach may be taken. Sort the distinct values of the observations on the threshold variable $q_{i t}$. Eliminate the smallest and largest $\eta \%$ for some $\eta>0$. The remaining $N$ values constitute the values of $\gamma$ which can be searched for $\hat{\gamma}$. For each of these $N$ values, regressions (6) are estimated yielding the sum of squared errors (7). The smallest value of the latter yields the estimate $\hat{\gamma}$. 
In practice, $N$ may be a very large number, and the optimization search describe above may be numerically intensive. A simplifying shortcut which yields nearly identical results is to restrict the search to a smaller set of values of $\gamma$. Instead of searching over all values of $q_{i t}$ (between the $\eta \%$ and $(1-\eta) \%$ quantile) the search may be limited to specific quantiles, perhaps integer valued. This greatly reduces the number of regressions performed in the search. The estimates from such an approximation are likely to be sufficiently precise for most applications of interest. For the empirical work reported in Section 4, we used the grid $\{1.00 \%, 1.25 \%, 1.50 \%, 1.75 \%, 2 \%, \ldots, 99.0 \%\}$ which contains 393 quantiles.

\section{Inference}

\subsection{Testing for a threshold}

It is important to determine whether the threshold effect is statistically significant. The hypothesis of no threshold effect in (1) can be represented by the linear constraint

$$
\mathrm{H}_{0}: \quad \beta_{1}=\beta_{2} .
$$

Under $\mathrm{H}_{0}$ the threshold $\gamma$ is not identified, so classical tests have non-standard distributions. This is typically called the 'Davies' Problem' (see Davies, 1977, 1987) and has been recently investigated by Andrews and Ploberger (1994) and Hansen (1996). The fixed-effects equations (4) fall in the class of models considered by Hansen (1996) who suggested a bootstrap to simulate the asymptotic distribution of the likelihood ratio test.

Under the null hypothesis of no threshold, the model is

$$
y_{i t}=\mu_{i}+\beta_{1}^{\prime} x_{i t}+e_{i t} .
$$

After the fixed-effect transformation is made, we have

$$
y_{i t}^{*}=\beta_{1}^{\prime} x_{i t}^{*}+e_{i t}^{*} .
$$

The regression parameter $\beta_{1}$ is estimated by OLS, yielding estimate $\widetilde{\beta}_{1}$, residuals $\tilde{e}_{i t}^{*}$ and sum of squared errors $S_{0}=\tilde{e}^{*} \tilde{e}^{*}$. The likelihood ratio test of $\mathrm{H}_{0}$ is based on

$$
F_{1}=\left(S_{0}-S_{1}(\hat{\gamma})\right) / \hat{\sigma}^{2} .
$$

The asymptotic distribution of $F_{1}$ is non-standard, and strictly dominates the $\chi_{k}^{2}$ distribution. Unfortunately, it appears to depend in general upon moments of the sample and thus critical values cannot be tabulated. Hansen (1996) shows 
that a bootstrap procedure attains the first-order asymptotic distribution, so p-values constructed from the bootstrap are asymptotically valid. ${ }^{3}$ Given the panel nature of the data we recommend the following implementation of the bootstrap. Treat the regressors $x_{i t}$ and threshold variable $q_{i t}$ as given, holding their values fixed in repeated bootstrap samples. Take the regression residuals $\hat{e}_{i t}^{*}$, and group them by individual: $\hat{e}_{i}^{*}=\left(\hat{e}_{i 1}^{*}, \hat{e}_{i 2}^{*}, \ldots, \hat{e}_{i T}^{*}\right)$. Treat the sample $\left\{\hat{e}_{1}^{*}, \hat{e}_{2}^{*}, \ldots, \hat{e}_{n}^{*}\right\}$ as the empirical distribution to be used for bootstrapping. Draw (with replacement) a sample of size $n$ from the empirical distribution and use these errors to create a bootstrap sample under $\mathrm{H}_{0}$. (Notice that the test statistic $F_{1}$ does not depend on the parameter $\beta_{1}$ under $\mathrm{H}_{0}$, so any value of $\beta_{1}$ may be used.) Using the bootstrap sample, estimate the model under the null (11) and alternative (4) and calculate the bootstrap value of the likelihood ratio statistic $F_{1}$ (12). Repeat this procedure a large number of times and calculate the percentage of draws for which the simulated statistic exceeds the actual. This is the bootstrap estimate of the asymptotic p-value for $F_{1}$ under $\mathrm{H}_{0}$. The null of no threshold effect is rejected if the p-value is smaller than the desired critical value.

\subsection{Asymptotic distribution of threshold estimate}

When there is a threshold effect $\left(\beta_{1} \neq \beta_{2}\right)$ Chan (1993) and Hansen (1999) have shown that $\hat{\gamma}$ is consistent for $\gamma_{0}$ (the true value of $\gamma$ ) and that the asymptotic distribution is highly non-standard. Hansen (1999) argues that the best way to form confidence intervals for $\gamma$ is to form the 'no-rejection region' using the likelihood ratio statistic for tests on $\gamma$. To test the hypothesis $\mathrm{H}_{0}: \gamma=\gamma_{0}$, the likelihood ratio test is to reject for large values of $L R_{1}\left(\gamma_{0}\right)$ where

$$
L R_{1}(\gamma)=\left(S_{1}(\gamma)-S_{1}(\hat{\gamma})\right) / \hat{\sigma}^{2} .
$$

Note that the statistic (13) is testing a different hypothesis from the statistic (12) introduced in the previous section. $L R_{1}\left(\gamma_{0}\right)$ is testing $\mathrm{H}_{0}: \gamma=\gamma_{0}$ while $F_{1}$ is testing $\mathrm{H}_{0}: \beta_{1}=\beta_{2}$.

Theorem 1. Under Assumptions 1-8 given in the Appendix, and $\mathrm{H}_{0}: \gamma=\gamma_{0}$,

$$
L R_{1}(\gamma) \rightarrow{ }_{d} \xi
$$

as $n \rightarrow \infty$, where $\xi$ is a random variable with distribution function

$$
\mathrm{P}(\xi \leqslant x)=(1-\exp (-x / 2))^{2} .
$$

\footnotetext{
${ }^{3}$ Since the asymptotic distribution is non-pivotal, bootstrap size will not have an accelerated rate of convergence relative to conventional asymptotic approximations. A referee suggested that pre-pivoting as in Beran (1987) may improve the convergence rate. This is an interesting suggestion and would be a constructive subject for future research.
} 
Theorem 1 shows that the asymptotic distribution of the likelihood ratio statistic is non-standard yet free of nuisance parameters. The technical assumptions include the rather unusual condition that $\left(\beta_{2}-\beta_{1}\right) \rightarrow 0$ as $n \rightarrow \infty$, and is borrowed from the changepoint literature. The condition means that the difference in the slopes between the two regimes is 'small' relative to sample size. Its practical relevance is that the asymptotic approximation implied by Theorem 1 is likely to hold better for cases where $\beta_{2}-\beta_{1}$ is small than for cases where $\beta_{2}-\beta_{1}$ is large. If the threshold effect is large, however, the threshold will be quite precisely estimated.

Since the asymptotic distribution in Theorem 1 is pivotal, it may be used to form valid asymptotic confidence intervals. Furthermore, the distribution function (14) has the inverse

$$
c(\alpha)=-2 \log (1-\sqrt{1-\alpha}),
$$

from which it is easy to calculate critical values. For example, the $10 \%$ critical value is 6.53 , the $5 \%$ is 7.35 and the $1 \%$ is 10.59 . A test of $\mathrm{H}_{0}: \gamma=\gamma_{0}$ rejects at the asymptotic level $\alpha$ if $L R_{1}\left(\gamma_{0}\right)$ exceeds $c(\alpha)$.

To form an asymptotic confidence interval for $\gamma$, the 'no-rejection region' of confidence level $1-\alpha$ is the set of values of $\gamma$ such that $L R_{1}(\gamma) \leqslant c(\alpha)$, where $L R_{1}(\gamma)$ is defined in (13) and $c(\alpha)$ is defined in (15). This is easiest to find by plotting $L R_{1}(\gamma)$ against $\gamma$ and drawing a flat line at $c(\alpha)$.

One of the convenient features of this confidence region is that it is a natural by-product of model estimation. In order to find the LS estimate $\hat{\gamma}$, the sequence of sum of squared errors $S_{1}(\gamma)$ were calculated. The likelihood ratio sequence $L R_{1}(\gamma)$ is a simple re-normalization of these numbers, and require no further computation.

\subsection{Asymptotic distribution of slope coefficients}

The estimator $\hat{\beta}=\widehat{\beta}(\hat{\gamma})$ depends on the threshold estimate $\hat{\gamma}$, which appears to complicate inference on $\beta$. Chan (1993) and Hansen (1999) show that the dependence on the threshold estimate is not of first-order asymptotic importance, so inference on $\beta$ can proceed as if the threshold estimate $\hat{\gamma}$ were the true value. Hence $\hat{\beta}$ is asymptotically normal with a covariance matrix which can be estimated by

$$
\hat{V}=\left(\sum_{i=1}^{n} \sum_{t=1}^{T} x_{i t}^{*}(\hat{\gamma}) x_{i t}^{*}(\hat{\gamma})^{\prime}\right)^{-1} \hat{\sigma}^{2} .
$$

While we need the assumption that the errors are iid for the purposes of constructing confidence intervals for $\gamma$, it would seem appropriate to relax this assumption when constructing confidence intervals for the slope coefficients. If 
the errors are allowed to be conditionally heteroskedastic, the natural covariance matrix estimator for $\hat{\beta}$ is

$$
\begin{aligned}
\hat{V}_{h}= & \left(\sum_{i=1}^{n} \sum_{t=1}^{T} x_{i t}^{*}(\hat{\gamma}) x_{i t}^{*}(\hat{\gamma})^{\prime}\right)^{-1}\left(\sum_{i=1}^{n} \sum_{t=1}^{T} x_{i t}^{*}(\hat{\gamma}) x_{i t}^{*}(\hat{\gamma})^{\prime}\left(\hat{e}_{i t}^{*}\right)^{2}\right) \\
& \times\left(\sum_{i=1}^{n} \sum_{t=1}^{T} x_{i t}^{*}(\hat{\gamma}) x_{i t}^{*}(\hat{\gamma})^{\prime}\right)^{-1} .
\end{aligned}
$$

\section{Multiple thresholds}

Model (1) has a single threshold. In some applications there may be multiple thresholds. For example, the double threshold model takes the form

$$
y_{i t}=\mu_{i}+\beta_{1}^{\prime} x_{i t} I\left(q_{i t} \leqslant \gamma_{1}\right)+\beta_{2}^{\prime} x_{i t} I\left(\gamma_{1}<q_{i t} \leqslant \gamma_{2}\right)+\beta_{3}^{\prime} x_{i t} I\left(\gamma_{2}<q_{i t}\right)+e_{i t}
$$

where the thresholds are ordered so that $\gamma_{1}<\gamma_{2}$. We will focus on this doublethreshold model since the methods extend in a straightforward manner to higher-order threshold models. We discuss three relevant statistical issues: (1) Estimation; (2) Testing for the presence of a double threshold; (3) Construction of confidence intervals for the threshold parameters $\gamma_{1}$ and $\gamma_{2}$.

\subsection{Estimation}

For given $\left(\gamma_{1}, \gamma_{2}\right),(16)$ is linear in the slopes $\left(\beta_{1}, \beta_{2}, \beta_{3}\right)$ so OLS estimation is appropriate. Thus for given $\left(\gamma_{1}, \gamma_{2}\right)$ the concentrated sum of squared errors $S\left(\gamma_{1}, \gamma_{2}\right)$ is straightforward to calculate (as in the single threshold model). The joint LS estimates of $\left(\gamma_{1}, \gamma_{2}\right)$ are by definition the values which jointly minimize $S\left(\gamma_{1}, \gamma_{2}\right)$. While these estimates might seem desirable, they may be quite cumbersome to implement in practice. A grid search over $\left(\gamma_{1}, \gamma_{2}\right)$ requires approximately $N^{2}=(n T)^{2}$ regressions which may be prohibitively expensive.

A remarkable insight allows us to escape this computational burden. It has been found (Chong, 1994; Bai, 1997; Bai and Perron, 1998) in the multiple changepoint model that sequential estimation is consistent. The same logic appears to apply to the multiple threshold model. The method works as follows. In the first stage, let $S_{1}(\gamma)$ be the single threshold sum of squared errors as defined in (7) and let $\hat{\gamma}_{1}$ be the threshold estimate which minimizes $S_{1}(\gamma)$. The analysis of Chong and Bai suggests that $\hat{\gamma}_{1}$ will be consistent ${ }^{4}$ for either $\gamma_{1}$ or $\gamma_{2}$ (depending on which effect is 'stronger').

\footnotetext{
${ }^{4}$ The reason why $\hat{\gamma}_{1}$ is consistent is because the single-threshold sum of squared errors function $S_{1}(\gamma)$ asymptotically converges to a limit function which has two local minima at $\gamma_{1}$ and $\gamma_{2}$.
} 
Fixing the first-stage estimate $\hat{\gamma}_{1}$, the second-stage criterion is

$$
S_{2}^{\mathrm{r}}\left(\gamma_{2}\right)= \begin{cases}S\left(\hat{\gamma}_{1}, \gamma_{2}\right) & \text { if } \hat{\gamma}_{1}<\gamma_{2} \\ S\left(\gamma_{2}, \hat{\gamma}_{1}\right) & \text { if } \gamma_{2}<\hat{\gamma}_{1}\end{cases}
$$

and the second-stage threshold estimate is

$$
\hat{\gamma}_{2}^{\mathrm{r}}=\operatorname{argmin} S_{2}^{\mathrm{r}}\left(\gamma_{2}\right) \text {. }
$$

Since it is undesirable to have a small number of observations in any given 'regime', we can restrict the search in (18) so that a minimum number of observations fall in each of the three regimes.

Bai (1997) has shown that $\hat{\gamma}_{2}^{\mathrm{r}}$ is asymptotically efficient, but $\hat{\gamma}_{1}$ is not. This is because the estimate $\hat{\gamma}_{1}$ was obtained from a sum of squared errors function which was contaminated by the presence of a neglected regime. The asymptotic efficiency of $\hat{\gamma}_{2}^{\mathrm{r}}$ suggests that $\hat{\gamma}_{1}$ can be improved by a third-stage estimation. Bai (1997) suggests the following refinement estimator. Fixing the second-stage estimate $\hat{\gamma}_{2}^{\mathrm{r}}$, define the refinement criterion

$$
S_{1}^{\mathrm{r}}\left(\gamma_{1}\right)= \begin{cases}S\left(\gamma_{1}, \hat{\gamma}_{2}^{\mathrm{r}}\right) & \text { if } \gamma_{1}<\hat{\gamma}_{2}^{\mathrm{r}}, \\ S\left(\hat{\gamma}_{2}^{\mathrm{r}}, \gamma_{1}\right) & \text { if } \hat{\gamma}_{2}^{\mathrm{r}}<\gamma_{1},\end{cases}
$$

and the refinement estimate

$$
\hat{\gamma}_{1}^{\mathrm{r}}=\underset{\gamma_{1}}{\operatorname{argmin}} S_{1}^{\mathrm{r}}\left(\gamma_{1}\right) \text {. }
$$

Bai (1997) shows that the refinement estimator $\hat{\gamma}_{1}^{\mathrm{r}}$ is asymptotically efficient in changepoint estimation, and we expect similar results to hold in threshold regression.

\subsection{Determining number of thresholds}

In the context of model (16), there are either no thresholds, one threshold, or two thresholds. In Section 3.1 we introduced $F_{1}$ as a test of no thresholds against one threshold, and suggested a bootstrap to approximate the asymptotic pvalue. If $F_{1}$ rejects the null of no threshold, in the context of model (16) we need a further test to discriminate between one and two thresholds.

The minimizing sum of squared errors from the second-stage threshold estimate is $S_{2}^{\mathrm{r}}\left(\hat{\gamma}_{2}^{\mathrm{r}}\right)$ with variance estimate $\hat{\sigma}^{2}=S_{2}^{\mathrm{r}}\left(\hat{\gamma}_{2}^{\mathrm{r}}\right) / n(T-1)$. Thus an approximate likelihood ratio test of one versus two thresholds can be based on the statistic

$$
F_{2}=\frac{S_{1}\left(\hat{\gamma}_{1}\right)-S_{2}^{\mathrm{r}}\left(\hat{\gamma}_{2}^{\mathrm{r}}\right)}{\hat{\sigma}^{2}}
$$


The hypothesis of one threshold is rejected in favor of two thresholds if $F_{2}$ is large.

Since the null asymptotic distribution of the likelihood ratio test is nonpivotal $^{5}$ we suggest using a bootstrap procedure to approximate the sampling distribution. To generate the bootstrap samples, hold the regressors $x_{i t}$ and threshold variable $q_{i t}$ fixed in repeated bootstrap samples. The bootstrap errors will be drawn from the residuals calculated under the alternative hypothesis, so should be the residuals from LS estimation of model (16). Group the regression residuals $\hat{e}_{i t}^{*}$ by individual: $\hat{e}_{i}^{*}=\left(\hat{e}_{i 1}^{*}, \hat{e}_{i 2}^{*}, \ldots, \hat{e}_{i T}^{*}\right)$, and treat the sample $\left\{\hat{e}_{1}^{*}, \hat{e}_{2}^{*}, \ldots, \hat{e}_{n}^{*}\right\}$ as an empirical distribution. Draw (with replacement) error samples from the empirical distribution. Let $e_{i}^{\#}$ denote a generic $T \times 1$ draw. The dependent variable $y_{i t}$ should be generated under the null hypothesis of a single threshold (1), so use the equation

$$
y_{i t}^{\#}=\widehat{\beta}_{1}^{\prime} x_{i t} I\left(q_{i t} \leqslant \hat{\gamma}\right)+\widehat{\beta}_{2}^{\prime} x_{i t} I\left(q_{i t}>\hat{\gamma}\right)+e_{i t}^{\#},
$$

which depends on the parameter values $\widehat{\beta}_{1}, \widehat{\beta}_{2}$, and $\hat{\gamma}$, the least-squares estimates from the single threshold model. From the bootstrap sample, the test statistic $F_{2}$ may be calculated, and this procedure repeated multiple times to calculate the bootstrap p-value.

From Eq. (21) it is clear that unlike the null sampling distribution of $F_{1}$, which asymptotically did not depend on $\gamma, \beta_{1}$ or $\beta_{2}$, the null sampling distribution of $F_{2}$ depends asymptotically on both $\gamma$ and the regression parameters $\beta_{1}$ and $\beta_{2}$, though it only depends on the latter through $\beta_{1}-\beta_{2}$. This leads us to expect that the bootstrap may not produce as accurate critical values for $F_{2}$ as for $F_{1}$, and neither is expected to be second-order accurate.

\subsection{Confidence region construction}

We finally consider the construction of confidence intervals for the two threshold parameters $\left\{\gamma_{1}, \gamma_{2}\right\}$. Bai (1997) showed (for the analogous case of change-point models) that the refinement estimators of Section 5.1 have the same asymptotic distributions as the threshold estimate in a single threshold model. This suggests that we can construct confidence intervals in the same way as in Section 4.2.

Let

$$
\operatorname{LR}_{2}^{\mathrm{r}}(\gamma)=\frac{S_{2}^{\mathrm{r}}(\gamma)-S_{2}^{\mathrm{r}}\left(\hat{\gamma}_{2}^{\mathrm{r}}\right)}{\hat{\sigma}^{2}}
$$

\footnotetext{
${ }^{5}$ It is important to remember that this differs from the changepoint case (see Chong, 1994, Bai, 1997; Bai and Perron, 1998), where the asymptotic distribution of $F_{2}$ is known and pivotal.
} 
and

$$
L_{1}^{\mathrm{r}}(\gamma)=\frac{S_{1}^{\mathrm{r}}(\gamma)-S_{1}^{\mathrm{r}}\left(\hat{\gamma}_{1}^{\mathrm{r}}\right)}{\hat{\sigma}^{2}}
$$

where $S_{2}^{\mathrm{r}}(\gamma)$ and $S_{1}^{\mathrm{r}}(\gamma)$ are defined in (17) and (19), respectively. Our asymptotic $(1-\alpha) \%$ confidence intervals for $\gamma_{2}$ and $\gamma_{1}$ are the set of values of $\gamma$ such that $L R_{2}^{\mathrm{r}}(\gamma) \leqslant c(\alpha)$ and $L R_{1}^{\mathrm{r}}(\gamma) \leqslant c(\alpha)$, respectively.

\section{Investment and financing constraints}

Classical models of the firm assume the existence of perfect financial markets on which firms can borrow the needed resources for investment projects. Alternative models of financing place restrictions on the extent of external financing. An important empirical question is whether or not there exist firms which behave as though they are subject to such constraints.

A well-cited paper which explored the empirical implications of financing constraints is Fazzari et al. (1988), henceforth FHP. These authors argue that the presence of financing constraints implies that a firm's cash flow will be positively related to its investment rate only when the firm faces constraints on external financing. If a firm is free to borrow on external financial markets, cash flow will be irrelevant for investment. This distinction motivated FHP to test for financing constraints by estimating separate investment regressions for 'constrained' and 'unconstrained' firms to see if there are differing effects of contemporaneous cash flow. To distinguish constrained and unconstrained firms, they used the dividend to income ratio, as their theory suggests that a financially constrained firm will choose to retain earnings rather than pay dividends. Hence the firms which have low levels of dividend payments are the financially constrained firms.

FHP divide their sample into three classes, depending on whether the dividend to income ratio was less than 0.1 for $10 \mathrm{yr}$ in the sample, between 0.1 and 0.2 for over $10 \mathrm{yr}$, and all other firms. Thus they are estimating a doublethreshold regression on panel data, where $q_{i t}$ is the largest dividend-income ratio over the 10-yr period, and the thresholds $\gamma$ are set at 0.1 and 0.2 .

There are two obvious problems with the FHP regression. First, it treats the dividend-income ratio as exogenous, while their theory explicitly treats dividend payments as decision variables. The use of an endogenous threshold variable may bias their results. Second, they select their threshold levels arbitrarily, rather than estimating these parameters from the sample. In this section we explore whether our methods allow for a re-appraisal of FHP's analysis.

The original data used by FHP is no longer available. We use a similar dataset, extracted from the dataset used by Hall and Hall (1993), which is an unbalanced panel of US firms originally taken from Compustat. Our methods 
are designed for balanced panels, so we took the subset of 565 firms which are observed for the years 1973-1987.

The threshold variable should be an exogenous indicator of a firm's access to external financing. A natural candidate is the existing debt level. It seems reasonable to believe that banks will be reluctant to lend money to debt-heavy firms. This choice is similar to that of $\mathrm{Hu}$ and Schiantarelli (1998) who estimate a switching regression using the debt-asset ratio as one variable in their switching equation.

To fix notation, let $I_{i t}$ be the ratio of investment to capital; $Q_{i t}$ be the ratio of total market value to assets; $C F_{i t}$ be the ratio of cash flow to assets; and $D_{i t}$ be the ratio of long-term debt to assets, where stock variables are defined at the end of year. Summary statistics of the four variables are given in Table 1.

We use the multiple threshold regression model

$$
\begin{aligned}
I_{i t}= & \mu_{i}+\theta_{1} Q_{i t-1}+\theta_{2} Q_{i t-1}^{2}+\theta_{3} Q_{i t-1}^{3}+\theta_{4} D_{i t-1} \\
& +\theta_{5} Q_{i t-1} D_{i t-1}+\beta_{1} C F_{i t-1} I\left(D_{i t-1} \leqslant \gamma_{1}\right) \\
& +\beta_{2} C F_{i t-1} I\left(\gamma_{1}<D_{i t-1} \leqslant \gamma_{2}\right)+\beta_{3} C F_{i t-1} I\left(\gamma_{2}<D_{i t-1}\right)+e_{i t},
\end{aligned}
$$

where (22) represents a double threshold model for illustration. Model (22) falls in the class of models (1) setting $q_{i t}=D_{i t-1}$ and $x_{i t}=C F_{i t-1}$. There are also the additional regressors $\left(Q_{i t-1}, Q_{i t-1}^{2}, Q_{i t-1}^{3}, D_{i t-1}, Q_{i t-1} D_{i t-1}\right)$. The latter can be viewed as a special case of (1) by constraining the slope coefficients on these variables to be the same in the two regimes, which has no effect on the distribution theory. The reason model (22) has only the slope coefficient on cash flow switch between regimes is to focus attention on this key variable of interest. The non-linear terms in the regression (namely, $Q_{i t-1}^{2}, Q_{i t-1}^{3}$, and $Q_{i t-1} D_{i t-1}$ ) were included to reduce the possibility of spurious correlations due to omitted variables bias. The choice of the particular non-linear terms was data-based, as the variables $D_{i t-1}^{2}$ and $D_{i t-1}^{3}$ were insignificant and omitted to reduce computation costs.

To determine the number of thresholds, model (22) was estimated by least squares, allowing for (sequentially) zero, one, two, and three thresholds. The test statistics $F_{1}, F_{2}$ and $F_{3}$, along with their bootstrap ${ }^{6}$ p-values, are shown in Table 2 . We find that the test for a single threshold $F_{1}$ is highly significant with a bootstrap p-value of 0.003 , and the test for a double threshold $F_{2}$ is also strongly significant, with a bootstrap p-value of 0.017 . On the other hand, the test for a third threshold $F_{3}$ is not close to being statistically significant, with a bootstrap p-value of 0.723 . We conclude that there is strong evidence that there are two thresholds in the regression relationship. For the remainder of the analysis we work with this double threshold model.

\footnotetext{
${ }^{6} 300$ bootstrap replications were used for each of the three bootstrap tests.
} 
Table 1

Summary statistics

\begin{tabular}{lclllc}
\hline & Minimum & $25 \%$ quantile & Median & $75 \%$ quantile & Maximum \\
\hline$I_{i t}$ & 0.001 & 0.049 & 0.076 & 0.113 & 1.66 \\
$Q_{i t-1}$ & 0.021 & 0.371 & 0.675 & 1.31 & 11.8 \\
$C F_{i t-1}$ & -0.94 & 0.12 & 0.22 & 0.32 & 8.71 \\
$D_{i t-1}$ & 0.000 & 0.089 & 0.206 & 0.320 & 4.67 \\
\hline
\end{tabular}

Table 2

Tests for threshold effects

\begin{tabular}{lc}
\hline Test for single threshold & \\
$F_{1}$ & 32.6 \\
P-value & 0.003 \\
$(10 \%, 5 \%, 1 \%$ critical values $)$ & $(12.4,14.8,26.2)$ \\
& \\
Test for double threshold & 25.8 \\
$F_{2}$ & 0.017 \\
P-value & $(12.3,14.9,42.9)$ \\
$(10 \%, 5 \%, 1 \%$ critical values $)$ & \\
Test for triple threshold & 4.2 \\
$F_{3}$ & 0.723 \\
P-value & $(10.9,13.3,22.9)$ \\
\hline
\end{tabular}

The point estimates of the two thresholds and their asymptotic $95 \%$ and $99 \%$ confidence intervals are reported in Table 3 . The estimates are 0.016 and 0.536 , which are very small (and very large) values in the empirical distribution of the debt/assets threshold variable. Thus the three classes of firms indicated by the point estimates are those with 'very low debt', 'very high debt' and 'other'. The asymptotic confidence intervals for the threshold are very tight, indicating little uncertainty about the nature of this division. More information can be learned about the threshold estimates from plots of the concentrated likelihood ratio function $L R_{1}(\gamma), L R_{2}^{\mathrm{r}}(\gamma)$ and $L R_{1}^{\mathrm{r}}(\gamma)$ in Figs. 1-3 (corresponding to the first-stage estimate $\hat{\gamma}_{1}$ and the refinement estimators $\hat{\gamma}_{2}^{\mathrm{r}}$ and $\left.\hat{\gamma}_{1}^{\mathrm{r}}\right)$. The point estimates are the value of $\gamma$ at which the likelihood ratio hits the zero axis, which is in the far left part of the graph. The $95 \%$ confidence intervals for $\gamma_{2}$ and $\gamma_{1}$ can be found from $L R_{2}^{\mathrm{r}}(\gamma)$ and $L R_{1}^{\mathrm{r}}(\gamma)$ by the values of $\gamma$ for which the likelihood ratio lies beneath the dotted line.

It is interesting to examine the unrefined first-step likelihood ratio function $L R_{1}(\gamma)$, which is computed when estimating a single threshold model. The 
Table 3

Threshold estimates

\begin{tabular}{llll}
\hline & Estimate & $95 \%$ confidence interval & $99 \%$ confidence interval \\
\hline$\hat{\gamma}_{1}^{\mathrm{r}}$ & 0.0157 & {$[0.0139,0.0181]$} & {$[0.0120,0.0239]$} \\
$\hat{\gamma}_{2}^{\mathrm{r}}$ & 0.5362 & {$[0.5305,0.5629]$} & {$[0.5190,0.5693]$} \\
\hline
\end{tabular}

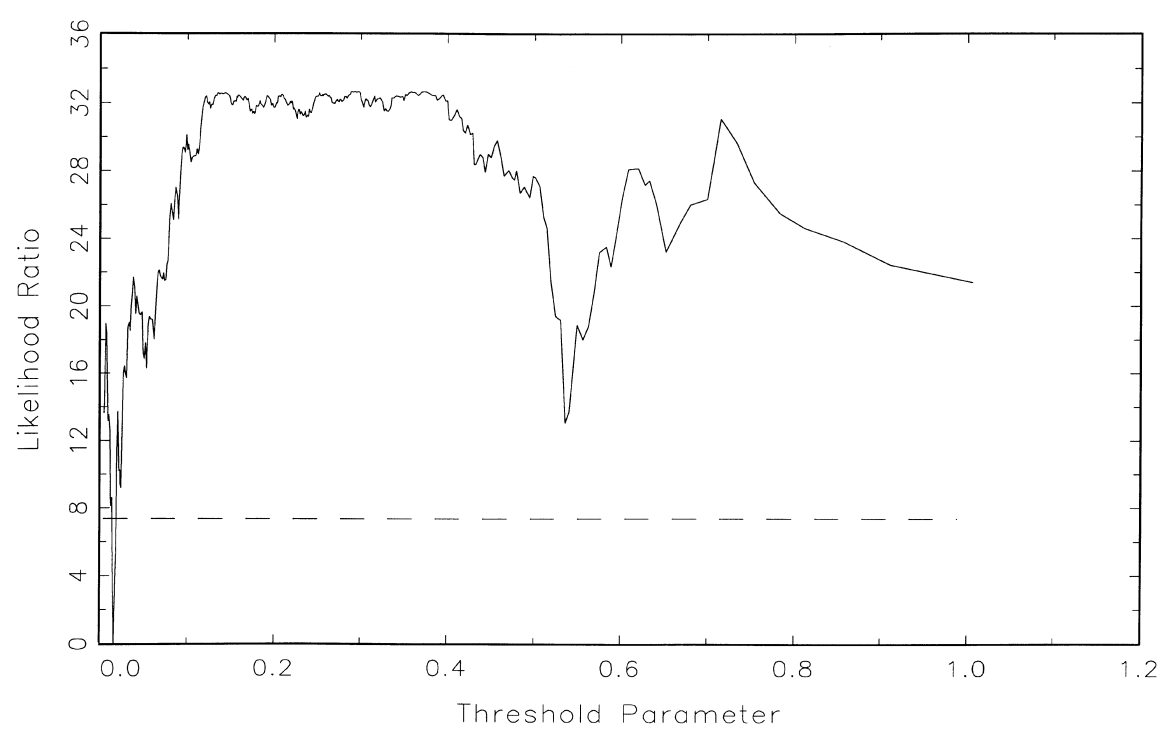

Fig. 1. Confidence interval construction in single threshold model.

first-step threshold estimate is the point where the $L R_{1}(\gamma)$ equals zero, which occurs at $\hat{\gamma}_{1}=0.0157$. There is a second major dip in the likelihood ratio around the second-step estimate 0.53 . Thus the single threshold likelihood conveys information that suggests that there is a second threshold in the regression.

Table 4 reports the percentage of firms which fall into the three regimes each year. We see that the percentage of firms in the 'very low debt' category ranges from $10 \%$ to $16 \%$ of the sample over the years. The 'very high debt' firms range from $4 \%$ to $16 \%$ of the sample in a given year. It is interesting to note that the last two years of the sample (1986 and 1987) saw a large increase in the number of firms with very high debt ratios.

The regression slope estimates, conventional OLS standard errors, and White-corrected standard errors are displayed in Table 5. We see that $Q_{i t-1}$ and its powers are statistically significant, indicating a positive (and very slightly 


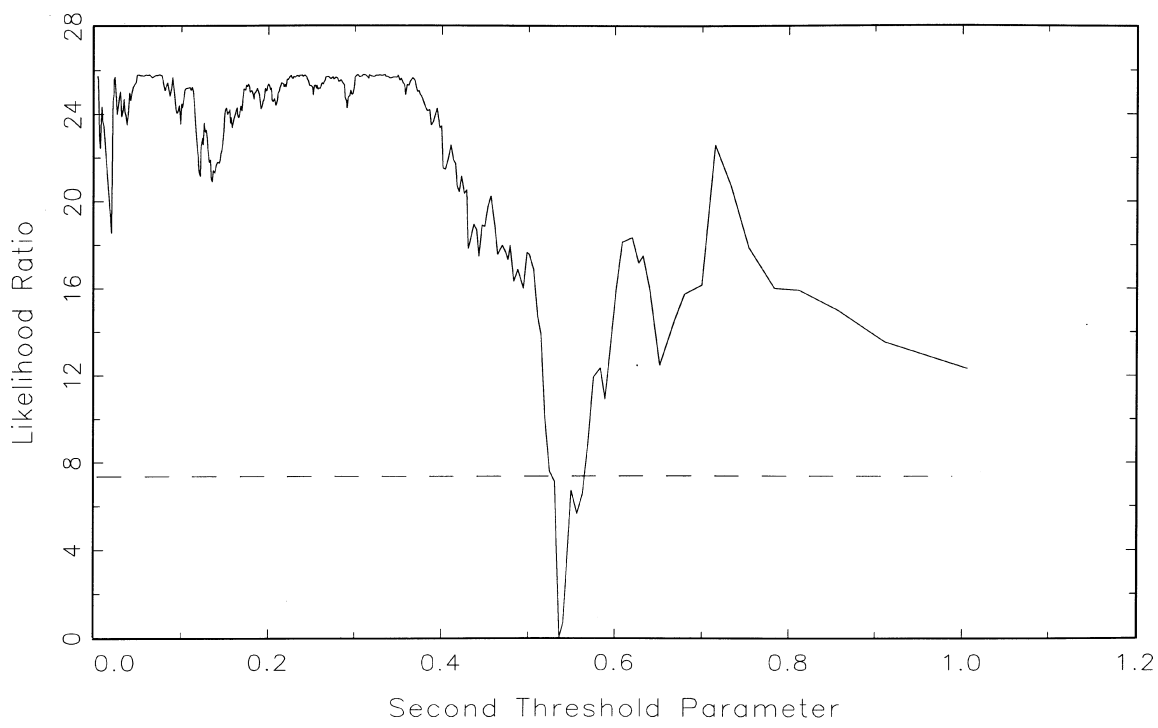

Fig. 2. Confidence interval construction in double threshold model.

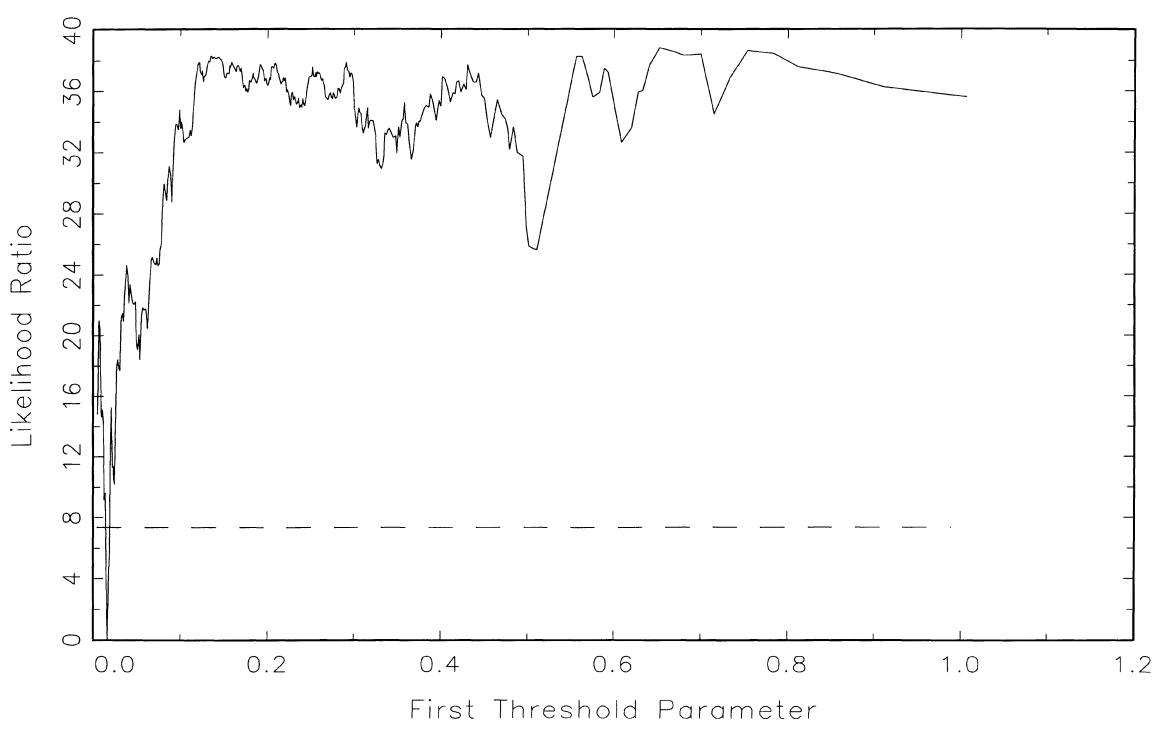

Fig. 3. Confidence interval construction in double threshold model.

non-linear) relationship between $q$ and investment. The debt level $D_{i t-1}$ has a negative and significant effect on investment, and there is no apparent interaction effect between $q$ and the debt level. 
Table 4

Percentage of firms in each regime by year

\begin{tabular}{lrrrrrrrrrrrrrr}
\hline \multicolumn{10}{c}{ Year } \\
\hline Firm class & 1974 & 1975 & 1976 & 1977 & 1978 & 1979 & 1980 & 1981 & 1982 & 1983 & 1984 & 1985 & 1986 & 1987 \\
\hline$D_{i t-1} \leqslant 0.0157$ & 16 & 14 & 14 & 15 & 15 & 13 & 13 & 11 & 10 & 10 & 10 & 10 & 10 & 11 \\
$0.0157<D_{i t-1} \leqslant 0.5362$ & 78 & 79 & 78 & 81 & 81 & 84 & 82 & 85 & 86 & 85 & 84 & 82 & 77 & 73 \\
$0.5362<D_{i t-1}$ & 6 & 7 & 8 & 5 & 4 & 4 & 5 & 4 & 4 & 5 & 6 & 8 & 13 & 16 \\
\hline
\end{tabular}

Table 5

Regression estimates: double threshold model

\begin{tabular}{lccc}
\hline Regressor & Coefficient estimate & OLS SE & White SE \\
\hline$Q_{i t-1}$ & 0.010 & 0.001 & 0.002 \\
$Q_{i t-1}^{2} / 10^{3}$ & -0.198 & 0.026 & 0.064 \\
$Q_{i t-1}^{3} / 10^{6}$ & 1.047 & 0.199 & 0.448 \\
$D_{i t-1}$ & -0.016 & 0.005 & 0.009 \\
$Q_{i t-1} D_{i t-1}$ & 0.001 & 0.001 & 0.002 \\
$C F_{i t-1} I\left(D_{i t-1} \leqslant 0.0157\right)$ & 0.063 & 0.006 & 0.014 \\
$C F_{i t-1} I\left(0.0157<D_{i t-1} \leqslant 0.5362\right)$ & 0.098 & 0.006 & 0.010 \\
$C F_{i t-1} I\left(0.5362<D_{i t-1}\right)$ & 0.039 & 0.012 & 0.031 \\
\hline
\end{tabular}

The coefficients of primary interest are those on cash flow. The point estimates suggest that investment is positively related to cash flow, with 'very low debt' firms having a lower coefficient (about one-third smaller in magnitude) than the typical firm. What is quite unexpected is that the firms with the highest debt levels have the smallest coefficient of 0.039 . The White standard error on this last coefficient, however, is quite high, indicating that there is still considerable uncertainty in the estimate.

The conventional OLS standard errors and the White-corrected standard errors are considerably different, with the White-corrected ones roughly twice as big. This is evidence in favor of heteroskedasticity, which violates one of the maintained assumptions of our asymptotic analysis. Based on the theory (Hansen, 1999) for least squares threshold regression (the model without fixed effects), we would expect the threshold estimates to be consistent and the distribution theory of Theorem 1 to be correct up to a scale effect, so that asymptotic confidence intervals would still take the form given in Table 3, but would require a different critical value. 


\section{Conclusion}

This paper has developed new empirical methods for panel data. We have defined a threshold regression model with individual-specific effects, and shown that the model is rather straightforward to estimate using a fixedeffects transformation. The asymptotic theory is non-standard, but confidence intervals for the threshold can be constructed by inverting the likelihood ratio statistic, and this construction is a natural by-product of the estimation method.

The methods are applied to the investment decisions of a panel of 565 US firms for the period 1973-1987. We find overwhelming evidence of a double threshold effect which separates the firms based on their debt to asset ratio. The estimates are somewhat consistent with the theory of financing constraints. The notable difference between our work and that of Fazzari et al. (1988) is that we are also able to quantify the extent of financing constraints in the economy rather than assuming the degree of such constraints.

Several extensions of our methods would be desirable, including allowing for heteroskedasticity, lagged dependent variables, endogenous variables, and random effects. It would also be interesting to compare our results with alternative approximations based on smooth transition threshold models, which replace the indicator functions by smooth distribution functions. These would be useful subjects for future research.

\section{Acknowledgements}

This research was supported by a grant from the National Science Foundation and a Sloan Foundation Research Fellowship. Special thanks go to Fabio Schiantarelli for many helpful discussions and motivation, to Richard Blundell for an insightful discussion, two referees for correcting some of my errors, and to Maria Laura Parisi for research assistance.

\section{Appendix. Mathematical proofs}

We need the following technical assumptions. Let $\gamma_{0}$ denote the true value of $\gamma$. Let $\theta=\beta_{2}-\beta_{1}$ and $C=n^{\alpha} \theta$, where $\alpha \in(0,1 / 2)$. Let $f_{t}(\gamma)$ denote the density function of $q_{i t}$, set $z_{i t}=C^{\prime} x_{i t}$,

$$
D(\gamma)=\sum_{t=1}^{T} \mathrm{E}\left(z_{i t}^{2} \mid q_{i t}=\gamma\right) f_{t}(\gamma),
$$

and $D=D\left(\gamma_{0}\right)$. Let $f_{k \mid t}\left(\gamma_{1} \mid \gamma_{2}\right)$ denote the conditional density of $q_{i k}$ given $q_{i t}$. 


\section{Assumptions}

1. For each $t,\left(q_{i t}, x_{i t}, e_{i t}\right)$ are independent and identically distributed (iid) across $i$.

2. For each $i, e_{i t}$ is iid over $t$, is independent of $\left\{\left(x_{i j}, q_{i j}\right)_{j=1}^{\mathrm{T}}\right\}$, and $\mathrm{E}\left(e_{i t}\right)=0$.

3. For each $j=1, \ldots, k, \mathrm{P}\left(x_{i 1}^{j}=x_{i 2}^{j}=\cdots=x_{i T}^{j}\right)<1$, where $x_{i t}^{j}$ is the $j$ th element of $x_{i t}$.

4. $\mathrm{E}\left|x_{i t}\right|^{4}<\infty$ and $\mathrm{E}\left|e_{i t}\right|^{4}<\infty$.

5. For some fixed $C<\infty$ and $0<\alpha<1 / 2, \theta=n^{-\alpha} C$.

6. $D(\gamma)$ is continuous at $\gamma=\gamma_{0}$.

7. $0<D<\infty$.

8. For $k>t, f_{k \mid t}\left(\gamma_{0} \mid \gamma_{0}\right)<\infty$.

Assumptions 1-4 are standard for fixed effect panel models with strictly exogenous regressors. Assumption 5 is more unusual, setting $\theta=n^{-\alpha} C \rightarrow 0$ as $n \rightarrow \infty$. The renormalization is to force $\theta$ to be 'small', reducing the information in the sample concerning the threshold and hence slowing down the rate of convergence of the threshold estimate. This assumption need not be viewed as very restrictive since the rate at which $\theta$ decreases to zero can be set quite low. It does suggest, however, that the asymptotic approximation is more likely to provide good approximations when $\theta$ is small relative to the case where $\theta$ is large.

Assumption 6 excludes threshold effects which occur simultaneously in the marginal distribution of the regressors and in the regression function. Assumption 7 excludes continuous threshold models (see Chan and Tsay, 1998), and requires that the threshold variable $q_{i t}$ be continuously distributed with positive support at the threshold $\gamma_{0}$. Assumption 8 excludes the possibility that $q_{i t}=\gamma_{0}$ for $t=1, \ldots, T$.

The proof of the theorem is based on the following lemma. Let ' $\Rightarrow$ ' denote weak convergence with respect to the uniform metric, and let $\lambda_{n}=n^{1-2 \alpha}$.

Lemma A.1. As $n \rightarrow \infty$, uniformly over $v \in[-\bar{v}, \bar{v}]$,

$$
S_{1}\left(\gamma_{0}\right)-S_{1}\left(\gamma_{0}+v / \lambda_{n}\right) \Rightarrow q(v)
$$

where

$$
q(v)=-D_{T}|v|+2 \sqrt{\sigma^{2} D_{T}} W(v)
$$

$D_{T}=D(1-1 / T)$, and $W(v)$ is a double-sided standard Brownian motion on $(-\infty, \infty)$.

Proof. Let

$$
\nabla z_{i t}(\gamma)=z_{i t} I\left(q_{i t} \leqslant \gamma\right)-z_{i t} I\left(q_{i t} \leqslant \gamma_{0}\right) .
$$


The regression equation (1) holds when $\gamma=\gamma_{0}$, the true value. For values of $\gamma \neq \gamma_{0}$, note that (1) can be re-written as

$$
\begin{aligned}
y_{i t}= & \mu_{i}+\beta_{1}^{\prime} x_{i t} I\left(q_{i t} \leqslant \gamma_{0}\right)+\beta_{2}^{\prime} x_{i t} I\left(q_{i t}>\gamma_{0}\right)+e_{i t} \\
= & \mu_{i}+\beta_{1}^{\prime} x_{i t} I\left(q_{i t} \leqslant \gamma\right)+\beta_{2}^{\prime} x_{i t} I\left(q_{i t}>\gamma\right) \\
& -\beta_{1}^{\prime} x_{i t}\left[I\left(q_{i t} \leqslant \gamma\right)-I\left(q_{i t} \leqslant \gamma_{0}\right)\right]-\beta_{2}^{\prime} x_{i t}\left[I\left(q_{i t}>\gamma\right)-I\left(q_{i t}>\gamma_{0}\right)\right]+e_{i t} \\
= & \mu_{i}+\beta^{\prime} x_{i t}(\gamma)+\left(\beta_{2}-\beta_{1}\right)^{\prime} x_{i t}\left[I\left(q_{i t} \leqslant \gamma\right)-I\left(q_{i t} \leqslant \gamma_{0}\right)\right]+e_{i t} \\
= & \mu_{i}+\beta^{\prime} x_{i t}(\gamma)+n^{-\alpha} \nabla z_{i t}(\gamma)+e_{i t} .
\end{aligned}
$$

Eq. (A.1) makes explicit the regression error for $\gamma \neq \gamma_{0}$.

The fixed effect transformation is linear, so can be applied to (A.1) to yield

$$
y_{i}^{*}=\beta^{\prime} x_{i t}^{*}(\gamma)+n^{-\alpha} \nabla z_{i t}^{*}(\gamma)+e_{i t}^{*}
$$

which is the correct representation of (4) for $\gamma \neq \gamma_{0}$. Hansen (1999) shows that the asymptotic distribution of $\hat{\gamma}$ is not affected by the estimation of $\beta$, and this holds in our environment as well. We can thus simplify matters by assuming that $\beta$ is known and only $\gamma$ is estimated, so that the regression residual (for fixed $\gamma$ ) is

$$
\hat{e}_{i t}(\gamma)=n^{-\alpha} \nabla z_{i t}^{*}(\gamma)+e_{i t}^{*}
$$

Using (A.2),

$$
\begin{aligned}
S\left(\gamma_{0}\right)-S(\gamma) & =\sum_{i=1}^{n} \sum_{t=1}^{T} \hat{e}_{i t}\left(\gamma_{0}\right)^{2}-\sum_{i=1}^{n} \sum_{t=1}^{T} \hat{e}_{i t}(\gamma)^{2} \\
& =\sum_{i=1}^{n} \sum_{t=1}^{T} e_{i t}^{* 2}-\sum_{i=1}^{n} \sum_{t=1}^{T}\left(n^{-\alpha} \nabla z_{i t}^{*}(\gamma)+e_{i t}^{*}\right)^{2} \\
& =-n^{-2 \alpha} \sum_{i=1}^{n} \sum_{t=1}^{T} \nabla z_{i t}^{*}(\gamma)^{2}-2 n^{-\alpha} \sum_{i=1}^{n} \sum_{t=1}^{T} \nabla z_{i t}^{*}(\gamma) e_{i t}^{*} .
\end{aligned}
$$

We now show that as $n \rightarrow \infty$, uniformly over $v \in[-\bar{v}, \bar{v}]$,

$$
n^{-2 \alpha} \sum_{i=1}^{n} \sum_{t=1}^{T} \nabla z_{i t}^{*}\left(\gamma_{0}+v / \lambda_{n}\right)^{2} \Rightarrow D_{T}|v|
$$

We prove (A.4) for the case $v \in[0, \bar{v}]$. We will show that for $\gamma=\gamma_{0}+v / \lambda_{n}$,

$$
\mathrm{E}\left(n^{-2 \alpha} \sum_{i=1}^{n} \sum_{t=1}^{T} \nabla z_{i t}^{*}(\gamma)^{2}\right)=\lambda_{n} \sum_{t=1}^{T} \mathrm{E}\left(\nabla z_{i t}^{*}(\gamma)\right)^{2} \rightarrow D_{T}|v| .
$$


Arguments similar to those in the proof of Lemma A.10 of Hansen (1999) show that (A.5) implies (A.4) under the assumptions. Expansion of the quadratic yields

$$
\sum_{t=1}^{T} \mathrm{E}\left(\nabla z_{i t}^{*}(\gamma)\right)^{2}=\sum_{t=1}^{T} \mathrm{E}\left(\nabla z_{i t}(\gamma)\right)^{2}-\frac{1}{T} \sum_{t=1}^{T} \sum_{k=1}^{T} \mathrm{E}\left(\nabla z_{i t}(\gamma) \nabla z_{i k}(\gamma)\right)
$$

Consider the first sum on the right-hand-side of (A.6). Observe that since $\gamma=\gamma_{0}+v / \lambda_{n} \rightarrow \gamma_{0}$

$$
\begin{aligned}
\lambda_{n} \mathrm{P}\left(\gamma_{0}<q_{i t} \leqslant \gamma\right) & =v \frac{\mathrm{P}\left(q_{i t} \leqslant \gamma\right)-\mathrm{P}\left(q_{i t} \leqslant \gamma_{0}\right)}{\gamma-\gamma_{0}} \\
& \rightarrow v f_{t}\left(\gamma_{0}\right)
\end{aligned}
$$

as $n \rightarrow \infty$. Thus

$$
\begin{aligned}
\lambda_{n} \sum_{t=1}^{T} \mathrm{E}\left(\nabla z_{i t}(\gamma)\right)^{2} & =\lambda_{n} \sum_{t=1}^{T} \mathrm{E}\left(z_{i t}^{2} I\left(\gamma_{0}<q_{i t} \leqslant \gamma\right)\right) \\
& =\sum_{t=1}^{T} \mathrm{E}\left(z_{i t}^{2} \mid \gamma_{0}<q_{i t} \leqslant \gamma\right) \lambda_{n} \mathrm{P}\left(\gamma_{0}<q_{i t} \leqslant \gamma\right) \\
& \rightarrow \sum_{t=1}^{T} v \mathrm{E}\left(z_{i t}^{2} \mid q_{i t}=\gamma_{0}\right) f_{t}\left(\gamma_{0}\right)=v D .
\end{aligned}
$$

Next consider the double-sum on the right-hand-side of (A.6). By Assumption 8 , for $k>t$,

$$
\begin{aligned}
\mathrm{P} & \left(\gamma_{0}<q_{i k} \leqslant \gamma \mid \gamma_{0}<q_{i t} \leqslant \gamma\right) \\
& =v \lambda_{n}^{-1} \frac{\left[\mathrm{P}\left(q_{i k} \leqslant \gamma \mid \gamma_{0}<q_{i t} \leqslant \gamma\right)-\mathrm{P}\left(q_{i k} \leqslant \gamma_{0} \mid \gamma_{0}<q_{i t} \leqslant \gamma\right)\right]}{\gamma-\gamma_{0}} \\
& =v \lambda_{n}^{-1}\left(f_{k \mid t}\left(\gamma_{0} \mid \gamma_{0}\right)+\mathrm{o}(1)\right) \\
& \rightarrow 0,
\end{aligned}
$$

and combined with (A.7), for $k>t$,

$$
\begin{aligned}
& \lambda_{n} \mathrm{P}\left(\gamma_{0}<q_{i t} \leqslant \gamma, \gamma_{0}<q_{i k} \leqslant \gamma\right) \\
& \quad=\lambda_{n} \mathrm{P}\left(\gamma_{0}<q_{i k} \leqslant \gamma\right) \mathrm{P}\left(\gamma_{0}<q_{i t} \leqslant \gamma \mid \gamma_{0}<q_{i k} \leqslant \gamma\right) \rightarrow 0 .
\end{aligned}
$$

Eq. (A.9) also holds for $k<t$ by symmetry. Hence

$$
\begin{aligned}
\lambda_{n} & \frac{1}{T} \sum_{t=1}^{T} \sum_{k=1}^{T} \mathrm{E}\left(\nabla z_{i t}(\gamma) \nabla z_{i k}(\gamma)\right) \\
\quad= & \frac{1}{T} \sum_{t=1}^{T} \sum_{k=1}^{T} \lambda_{n} \mathrm{E}\left(z_{i t} z_{i k} I\left(\gamma_{0}<q_{i t} \leqslant \gamma\right) I\left(\gamma_{0}<q_{i k} \leqslant \gamma\right)\right)
\end{aligned}
$$




$$
\begin{aligned}
= & \frac{1}{T} \sum_{t=1}^{T} \sum_{k=1}^{T} \mathrm{E}\left(z_{i t}^{2} \mid \gamma_{0}<q_{i t} \leqslant \gamma, \gamma_{0}<q_{i k} \leqslant \gamma\right) \\
& \cdot \lambda_{n} \mathrm{P}\left(\gamma_{0}<q_{i t} \leqslant \gamma, \gamma_{0}<q_{i k} \leqslant \gamma\right) \\
= & \frac{1}{T} \sum_{t=1}^{T} \mathrm{E}\left(z_{i t}^{2} \mid \gamma_{0}<q_{i t} \leqslant \gamma\right) \lambda_{n} \mathrm{P}\left(\gamma_{0}<q_{i t} \leqslant \gamma\right)+\mathrm{o}(1) \\
\rightarrow & \frac{1}{T} \sum_{t=1}^{T} \mathrm{E}\left(z_{i t}^{2} \mid q_{i t}=\gamma_{0}\right) v f_{t}\left(\gamma_{0}\right)=\frac{1}{T} v D .
\end{aligned}
$$

by (A.9). Eqs. (A.6), (A.8) and (A.10) imply (A.5) and hence (A.4). Next, we wish to show that uniformly over $v \in[-\bar{v}, \bar{v}]$,

$$
n^{-\alpha} \sum_{i=1}^{n} \sum_{t=1}^{T} \nabla z_{i t}^{*}\left(\gamma_{0}+v / \lambda_{n}\right) e_{i t}^{*} \Rightarrow \sqrt{\sigma^{2} D_{T}} W(v) .
$$

By the properties of least squares projection, $\sum_{t=1}^{T} \nabla z_{i t}^{*}(\gamma) e_{i t}^{*}=\sum_{t=1}^{T} \nabla z_{i t}^{*}(\gamma) e_{i t}$, and since the $e_{i t}$ are iid,

$$
\begin{aligned}
\mathrm{E}\left(n^{-\alpha} \sum_{i=1}^{n} \sum_{t=1}^{T} \nabla z_{i t}^{*}(\gamma) e_{i t}^{*}\right)^{2} & =\lambda_{n} \mathrm{E}\left(\sum_{t=1}^{T} \nabla z_{i t}^{*}(\gamma) e_{i t}\right)^{2} \\
& =\lambda_{n} \sum_{t=1}^{T} \mathrm{E}\left(\nabla z_{i t}^{*}(\gamma)\right)^{2} \sigma^{2} \\
& \rightarrow D_{T}|v| \sigma^{2}
\end{aligned}
$$

by (A.5). This establishes that the finite dimensional distributions of the stochastic process are those of the stated double-sided Brownian motion. By arguments identical to those in the proof of Lemma A.11 of Hansen (1999), (A.12) and Assumption 1 are sufficient to establish (A.11). Eqs. (A.4) and (A.11) combine with (A.3) to yield the stated result.

Proof of Theorem 1. Since $\hat{\gamma}$ minimizes $S(\gamma)$,

$$
\begin{aligned}
L R_{1}\left(\gamma_{0}\right) & =\max _{\gamma}\left(\frac{S_{1}\left(\gamma_{0}\right)-S_{1}(\gamma)}{\hat{\sigma}^{2}}\right) \\
& =\max _{v}\left(\frac{S_{1}\left(\gamma_{0}\right)-S_{1}\left(\gamma_{0}+v / \lambda_{n}\right)}{\hat{\sigma}^{2}}\right),
\end{aligned}
$$

where the final equality makes the change-of-variables $\gamma=\gamma_{0}+v / \lambda_{n}$, Hansen (1999) shows that under Assumption 1,

$$
\hat{v} \equiv \lambda_{n}\left(\hat{\gamma}-\gamma_{0}\right)=\mathrm{O}_{\mathrm{p}}(1)
$$


We will not repeat the proof of (A.14) here. The stochastic boundedness of (A.14) shows that for any $\eta>0$, there is some $\bar{v}<\infty$ such that

$$
\mathrm{P}(|\hat{v}| \leqslant \bar{v}) \geqslant 1-\eta \text {. }
$$

Let

$$
\begin{aligned}
\widetilde{L R} & =\max _{|v| \leqslant \bar{v}}\left(\frac{S_{1}\left(\gamma_{0}\right)-S_{1}\left(\gamma_{0}+v / \lambda_{n}\right)}{\hat{\sigma}^{2}}\right) \\
& \Rightarrow \sigma^{-2} \max _{|v| \leqslant \bar{v}} q(v)
\end{aligned}
$$

where the stated weak convergence follows from Lemma A.1 and the continuous mapping theorem. Eq. (A.15) shows that

$$
\mathrm{P}\left(L R_{1}\left(\gamma_{0}\right)=\widetilde{L R}\right) \geqslant 1-\eta .
$$

Since $\eta$ is arbitrary we conclude that

$$
L R_{1}\left(\gamma_{0}\right) \Rightarrow \sigma^{-2} \max _{-\infty<v<\infty} q(v)=\xi
$$

say. Hansen (1999, Proof of Theorem 2) shows that the distribution function of $\xi$ is $\mathrm{P}(\xi \leqslant x)=(1-\exp (-x / 2))^{2}$.

\section{References}

Abel, A.B., Eberly, J.C., 1994. A unified model of investment under uncertainty. American Economic Review 84, 1369-1384.

Abel, A.B., Eberly, J.C., 1996. Investment and $q$ with fixed costs: An empirical analysis. Working paper, University of Pennsylvania.

Andrews, D.W.K., Ploberger, W., 1994. Optimal tests when a nuisance parameter is present only under the alternative. Econometrica 62, 1383-1414.

Bai, J., 1997. Estimating multiple breaks one at a time. Econometric Theory 13, 315-352.

Bai, J., Perron, P., 1998. Estimating and testing linear models with multiple structural changes. Econometrica 66, 47-78.

Barnett, S.A., Sakellaris, P., 1998. Non-linear response of firm investment to q: Testing a model of convex and non-convex adjustment costs. Journal of Monetary Economics 42, 261-288.

Beran, R., 1987. Prepivoting to reduce the level error of confidence sets. Biometrika 74, 457-468.

Chan, K.S., 1993. Consistency and limiting distribution of the least squares estimator of a threshold autoregressive model. The Annals of Statistics 21, 520-533.

Chan, K.S., Tsay, R.S., 1998. Limiting properties of the least squares estimator of a continuous threshold autoregressive model. Biometrika 85, 413-426.

Chong, T. T-L., 1994. Consistency of change-point estimators when the number of change-points in structural change models is underspecified. Working paper, Chinese University of Hong Kong.

Davies, R.B., 1977. Hypothesis testing when a nuisance parameter is present only under the alternative. Biometrika 64, 247-254.

Davies, R.B., 1987. Hypothesis testing when a nuisance parameter is present only under the alternative. Biometrika 74, 33-43. 
Fazzari, S.M., Glenn Hubbard, R., Petersen, B.C., 1988. Financing constraints and corporate investment. Brookings Papers on Economic Activity. pp. 141-195.

Hall, B.H., Hall, R.E., 1993. The value and performance of U.S. corporations. Brookings Papers on Economic Activity. pp. 1-34.

Hansen, B.E., 1996. Inference when a nuisance parameter is not identified under the null hypothesis. Econometrica 64, 413-430.

Hansen, B.E., 1999. Sample splitting and threshold estimation. Econometrica, forthcomming.

$\mathrm{Hu}, \mathrm{X}$., Schiantarelli, F., 1998. Investment and capital market imperfections: A switching regression approach using U.S. firm panel data. Review of Econometrics and Statistics 80, 466-479. 